\title{
Effect of indoor wheelchair curling training on trunk control of person with chronic spinal cord injury: a randomised controlled trial
}

\author{
Tanja Herzog ${ }^{1} \cdot$ Jaap Swanenburg $\mathbb{D}^{2,3} \cdot$ Markus Hupp ${ }^{1} \cdot$ Anne-Gabrielle Mittaz Hager ${ }^{4}$
}

Received: 14 November 2017 / Revised: 9 February 2018 / Accepted: 9 February 2018

(C) International Spinal Cord Society 2018

\begin{abstract}
Study design Randomised, controlled, single-blind crossover design study.

Objective Effect of indoor wheelchair curling training on trunk control of a person with chronic spinal cord injury (SCI). Setting SCI Centre of Balgrist University Hospital in Zurich, Switzerland.

Methods The trunk control of 13 subjects was assessed by the modified functional reach test (MFRT) and nonlinear dynamic systems analysis (NDSA) before and after eight indoor curling training sessions and compared to everyday life over 4 weeks.

Results The attendance rate was $95 \%$ during the training sessions. There were no adverse events. Neither the MRFT nor the NDSA showed any significant differences in the sitting stability. The subjects subjectively reported improved physical feeling, an increase in their trunk control and strength; 39\% of the participants wanted to continue the training.

Conclusions With subjective improvements and no adverse events, indoor wheelchair curling training is a safe physical activity for people with SCI. Wheelchair curling offers a suitable alternative to sports already used in rehabilitation and in recreational activities of a person with SCI. In order to be able to draw more unambiguous conclusions from the training method for trunk control and to answer the question of the study unambiguously, the number of subjects would have to be greater and the test methods should provide more exact and specific measurements.
\end{abstract}

\section{Introduction}

Spinal cord injury (SCI) is a serious life change, which occurs suddenly and unexpected with devastating consequences for a person's life [1]. Prevalence of SCI is estimated between 1:1000 persons per million inhabitants [2].

$\triangle$ Tanja Herzog

tanja.herzog@balgrist.ch

1 Spinal Cord Injury Center, University Hospital Balgrist, Zurich, Switzerland

2 Interdisciplinary Spinal Research ISR, Department of Chiropractic Medicine, Balgrist University Hospital, Zurich, Switzerland

3 Physiotherapy and Occupational Therapy Research Center, Directorate of Research and Education, University Hospital Zurich, Zurich, Switzerland

4 Domain Health and Social Work, University of Applied Sciences Valais, Leukerbad, Switzerland
Sequelae of SCI are loss of voluntary motor function, sensitivity and proprioception, resulting in a severe disruption in sitting stability. To improve sitting balance, resources like visual control, vestibular and cerebellar systems and remaining sensory and motor functions are utilised for compensation [3]. Maintaining postural control is a great challenge for persons with SCI [4]. Therefore, depending on the injury height [5] and the severity [6] of SCI, reaching movements and inadequate sitting stability may lead to falls with severe consequences like fractures. Therefore, good sitting stability is important in activities of daily life in wheelchair dependent patients. Accordingly, substantial time in rehabilitation programmes is devoted to improve sitting balance. One integral part is sports lessons, such as basketball, table tennis or archery, and kayaking [7]. Training must be effective and motivating for the patients. However, there is no standardised guideline for physiotherapy management for persons with paraplegia [8].

An attractive way for balance training is wheelchair curling [9]. The stones and the ice rink are equal to conventional curling, only players are seated in wheelchairs and there is no sweeping. It is allowed to release the stone by 
hand or with a telescopic stick. It is possible, with the newly developed wooden curling stones, to play the sport in a gymnastics hall instead of on a cold ice rink.

Successful players have good upper-body coordination and arm push force as well as a high tolerance for cold [10]. The latter is often lacking due to impairment of thermoregulation after SCI [11]. The objective of this study is to investigate the effect of indoor wheelchair curling training on trunk control in persons with chronic SCI.

\section{Methods}

\section{Participants}

Subjects with traumatic and non-traumatic SCI were recruited in the physiotherapy department at Balgrist University Hospital in Zurich, Switzerland. All subjects were participants visited the out-patient department at the hospital between June and September 2015. Injuries were assessed by International Standards for Neurological Classification of Spinal Cord Injury (ISNCSCI) [6]. The derived American spinal cord injury association (ASIA) Impairment Scale (AIS) had to be stable for at least 6 months. In addition, a duration of sitting in the wheelchair of at least $4 \mathrm{~h}$ and a position in which the upper body was capable of leaning forward by at least $20^{\circ}$ was necessary. Minimum age was 18 years. Exclusion criteria were progressive SCI pathologies, known dysfunction of vestibular system, severe visual restrictions, acute pain problems and restricted arm or hand function. Seventeen subjects with chronic SCI were screened for inclusion. Three persons declined to participate for personal reasons. One subject was subsequently excluded, because he had to be admitted to an inpatient setting due to disease-related complications. The measurements and the training sessions were conducted with 13 patients, who fulfilled all inclusion criteria. All participants signed a written informed consent. The study was approved by the Ethics Committee of the Canton of Zurich (KEK-ZH-Nt.2015-0161) and registered ClinicalTrials.gov Identifier:NCT02550834

\section{Study design}

The investigation was designed as a randomised, controlled, single-blind crossover study. Due to the expected small number of possible subjects, a crossover design was performed [12]. The subjects $(n=13)$ were randomised by an independent person in two groups. The breakdown was made by lot. Six subjects were attributed to the Group A and seven to non-training Group B. While Group A completed 4 weeks of training first, Group B did no extra training. After the first 4 weeks, the groups switched, with
Group B now on training and Group A without extra training. There were three measurement time points. The first at the start, the second at switch and the last at the end. The order of the measurements were at random. An experienced assessor with several years of experience took all measurements. The assessor was blinded to the group assignment. The pause between the assessments was determined individually. The participants were instructed to pause for as long as needed to feel refreshed for the next assessment. The intervention was conducted by two experienced physiotherapists. Both were trained in wheelchair curling.

\section{Intervention}

Indoor wheelchair curling was played as a training intervention. With the newly designed wooden curling stones, which slide over the floor with rollers affixed to the underbody, it was possible to play in a gymnastics hall. To release the stones telescopic sticks were provided which are also used in wheelchair curling on ice. A 10-metre-long carpet made of PVC with a pre-printed playing field served as the playing surface (Fig 1).

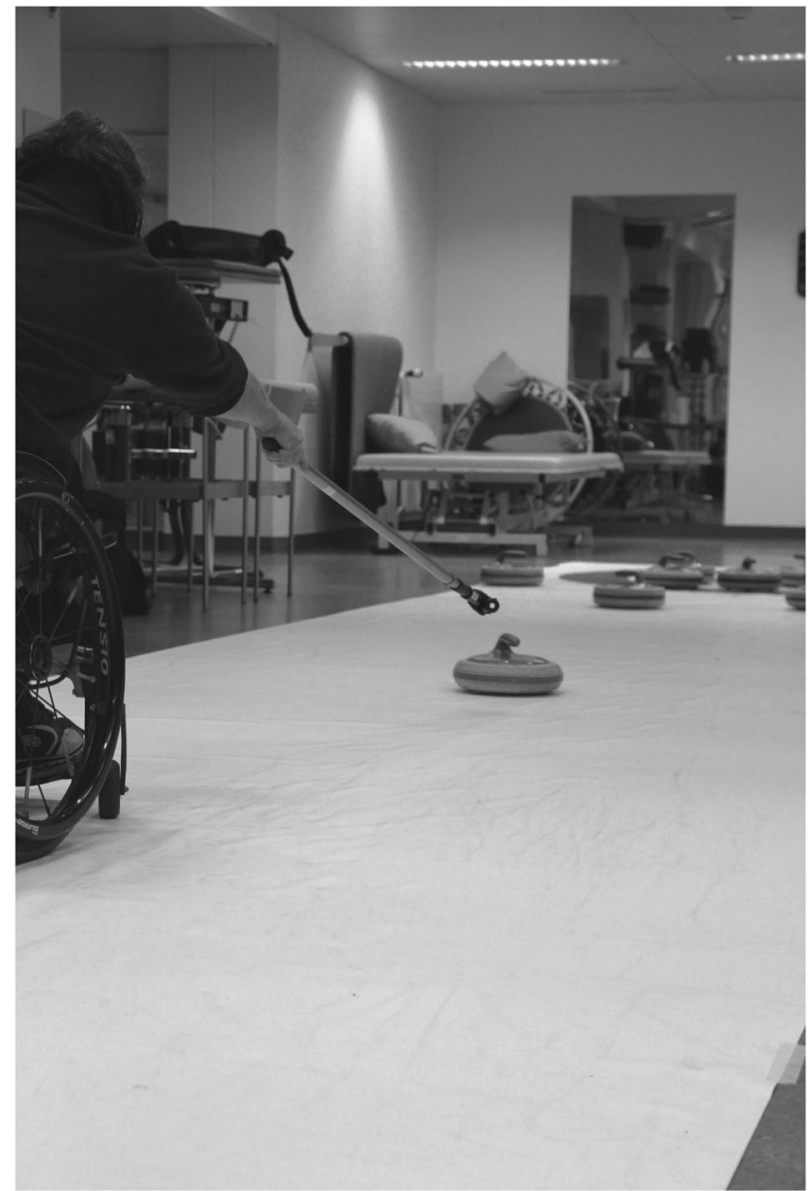

Fig. 1 Indoor wheelchair curling training 


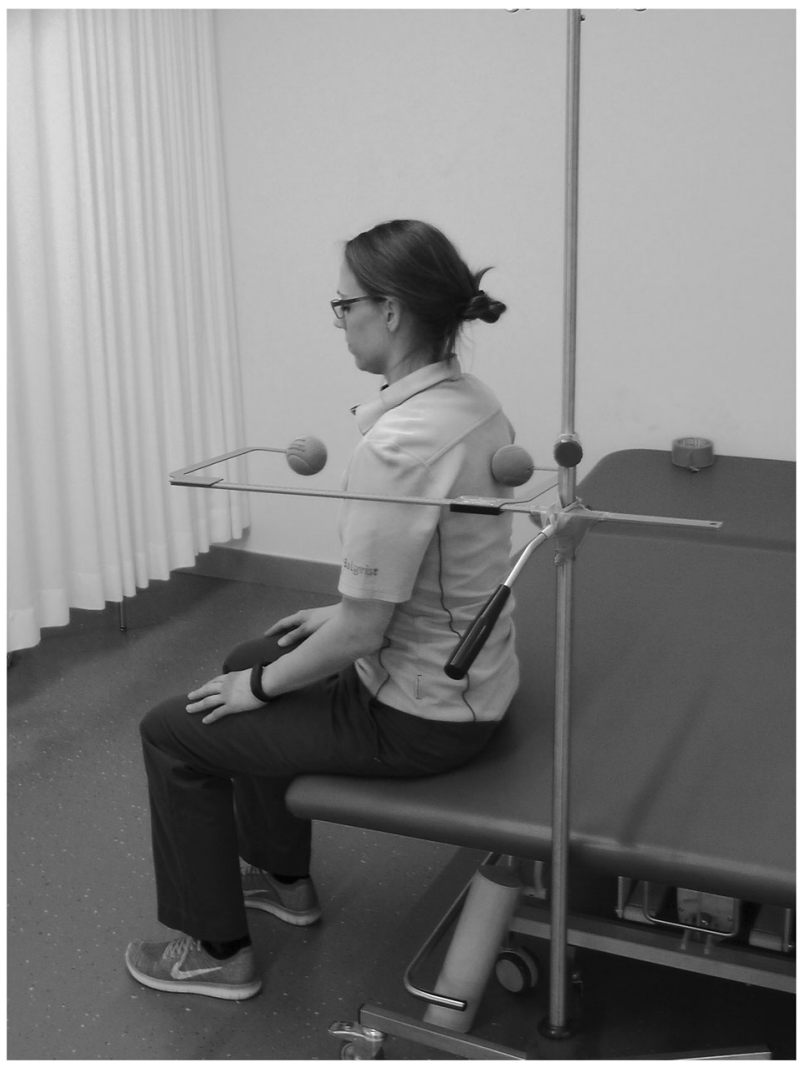

Fig. 2 Setup for Nonlinear Dynamic Systems Analysis (NDSA)

\section{Curling rules}

Rules are according to the World Curling Federation protocol [13]. In this study, the rules of wheelchair curling on ice were adopted, with the exception of the securing of the wheelchair by a fellow player. All participants used the telescopic stick to release the stone. Each participant completed two training session per week, amounting to eight training session in 4 weeks. Generally, one training unit lasted $90 \mathrm{~min}$ and involved a warm-up period $(10 \mathrm{~min})$, a main part with technical training $(30 \mathrm{~min})$ and playing (40 $\mathrm{min})$ as well as a cool down (10 $\mathrm{min})$. Attendance at the training sessions was recorded. During the study, the subjects in the control group were asked to pursue their normal habits and to continue their customary sporting activities or their therapy programme.

\section{Measurement instruments}

\section{Primary measurement parameter}

Modified functional reach test (MFRT) To measure the sitting balance, the MFRT [14] was used. The maximum distance that the subject was able to reach with his arm extended forward or sideward without losing his secure sitting position was measured. The tip of the middle finger was used as a reference. While making the movement, the patient sat on a therapy bench with knees and hips at a $90^{\circ}$ angle. For the movement the participant was asked to reach his hand as far forward as possible without supporting himself, rotating his upper body or losing his balance. The arm that was not used for the movement was allowed to remain on the upper leg without a supporting function. One training run and three assessments were made. The mean of the three assessments was counted.

\section{Secondary measurement parameters}

Nonlinear dynamic systems analysis (NDSA) In this study, the NDSA [15] was used to quantify neuromuscular control of the spinal stability, which was shown to be viable in a person with SCI [16]. The subject sat on a physio bench with knees and hips at a $90^{\circ}$ angle (Fig. 2), feet placed on the floor. A 3D accelerometer and gyroscope (Physilog, GaitUp, Lausanne, Switzerland) was attached to the patient's chest with an elastic band.

The subject performed a rhythmic forward and backward movement with his upper body. A rate of 25 repetitions per minute was pre-set using a metronome. The movement was performed from an upright position at $0^{\circ}$ to a forward tilt of $20^{\circ}$. Measurements were made twice for $1 \mathrm{~min}$ with a pause of $2 \mathrm{~min}$ between the tests. The first and last 5 measurements were not, but the remaining 15 movements were evaluated for the study. Calculations were based on the mean value of the two test attempts. The anterior-posterior acceleration (acc) and the angular acceleration around the mediolateral axis (rot) were analysed. Spectral analysis (SA) represents the irregularity in the movements. The local dynamic stability (LDS) shows to what extent the motor control ability is present to perform a smooth and constant movement.

Spinal cord independence measure (SCIM) For this study, the german translation of SCIM III [17] as a functional measurement of acitivities of daily living was used.

Open question The open question: "What other comments would you like to make about this study?" was asked at the conclusion of the study. In cases where the answers were unclear, more detailed questions were asked.

\section{Statistics}

Due to the small sample size, non-parametric testing was used. The presence of a carry-over effect was checked with the Mann-Whitney $U$-test. With no carry-over effect, groups $\mathrm{A}$ and $\mathrm{B}$ were combined for further analysis. To test 
the effect of the curling exercise a Wilcoxon-Rank-Test was used. The significance level of the statistical analysis was set at $p<0.05$.

The raw data from the accelerometer was analysed with the statistics programme STATA (Stata Corporation, 13). Further processing and statistical analyses were performed with SPSS 21 (IBM, PASW Statistics, Chicago, IL). The collection and storing of the data were made in REDCap (8.2.0, Vanderbilt University).

\section{Results}

Of the 13 participants, nine attended all eight training sessions. Three subjects had to cancel one training session ( $88 \%$ attendance) and one subject was not able to attend two training sessions (75\% attendance). The average attendance of all participants was $95 \%$. There were no adverse events reported. The participants' characteristics are summarised in in Table 1.

\section{Carry-over testing}

The MFRT and NDSA mean values and standard deviations of the 13 evaluated participants are reported in Table 2. One NDSA measurement of Group B could not be used due recording problem. The Mann-Whitney $U$-tests showed no significant difference and thus no carry-over effect of the MFRT forward $(p=0.498)$ and sideward $(p=0.477)$. Therefore, the data of Group A and B were pooled. The NDSA SA did not show a carry-over effect $\left(\mathrm{SA}_{\mathrm{acc}} p=\right.$ 0.093 and $\mathrm{SA}_{\text {rot }} p=.981$ ). For the analysis of $\mathrm{SA}_{\text {acc }}$ and

Table 1 Characteristics of the participants $(n=13)$

\begin{tabular}{lll}
\hline & Number $(\%)$ & Mean \pm SD \\
\hline Age & & $52 \pm 15.8$ \\
Size & & $170 \pm 7.8$ \\
Weight $(\mathrm{kg})$ & & $70 \pm 12.1$ \\
Sex & & \\
$\quad$ Female & $6(46.2)$ & \\
$\quad$ Male & $7(53.8)$ & \\
Injury & & \\
$\quad$ Traumatic & $8(61.5)$ & \\
$\quad$ Non-traumatic & $5(38.5)$ & \\
AIS & & \\
A & $4(30.8)$ & \\
B & $1(7.7)$ & \\
C & $4(30.8)$ & \\
D & $4(30.8)$ & \\
\hline
\end{tabular}

$S D$ standard, AIS American Spinal Injury Association Impairment Scale
$\mathrm{SA}_{\text {rot }}$ Groups A and B were pooled. No carry-over effect was found in the local dynamic stability $\operatorname{LDS}_{\text {acc }}(p=0.065)$ and $\operatorname{LDS}_{\text {rot }}(p=0.394)$. Therefore, the data of Group A and $\mathrm{B}$ were pooled.

\section{Effect testing}

A Wilcoxon signed-rank test showed that the curling intervention did not elicit a statistically significant change in MFRT forward and sideward. Indeed, both medians increased. The NDSA SA tests did not show any change with the Wilcoxon signed-rank test. All results are presented in Table 2.

\section{SCIM}

The SCIM did not show any difference between the measurement time points for all of the participants. The subjects of Group A received between 52 and 97 points and those in Group B between 55 and 100 points.

\section{Open question}

The results of the open question at the conclusion of the study are presented in Table 3. Two of the 13 participants did not have any comments. All comments were positive.

\section{Discussion}

The objective of this project was to investigate the effect of indoor wheelchair curling on trunk control in persons with chronic SCI. This is the first randomised study to investigate an effect of indoor curling on sitting stability and trunk control in wheelchair users.

The modified functional reach test (MFRT) is suited to test trunk control in wheelchair patients [14]. The results are comparable with outer studies [14, 18]. Nevertheless, the results showed no improvement in reaching out.

The nonlinear dynamic systems analysis (NDSA) is also an applicable assessment to measure trunk control in persons with SCI [16]. This study could only show a tendency toward improvement. The NDSA showed a high standard deviation in all results, implying a large variation in the data. However, difficulty in fine-tuning of the trunk was observed comparable to our study [16]. As a consequence, it was more difficult for persons with SCI compared to healthy subjects to gently touch the fixed point in front and behind. This may explains the large standard deviations in the former [16] and our study. This means that it is difficult to show evidence of an improvement in the trunk control by means of the NDSA, especially in a small population. In addition, the NDSA is still little known in healthy subjects 
Table 2 Results of the trunk control bevor and after indoor wheelchair curling training

\begin{tabular}{|c|c|c|c|c|c|c|}
\hline & \multicolumn{2}{|l|}{ Pretest } & \multicolumn{2}{|c|}{ Posttest $N=13$} & \multicolumn{2}{|c|}{ Wilcoxon-Rank-Test } \\
\hline & Mean \pm SD & Median & Mean \pm SD & Median & $z$ & $p$ \\
\hline \multicolumn{7}{|l|}{ MFRT } \\
\hline Forward $(n=13)$ & $31.4 \pm 12.6$ & 29.3 & $34.1 \pm 18.6$ & 32.7 & -1.22 & 0.22 \\
\hline Sideward $(n=13)$ & $15.8 \pm 5.2$ & 16.3 & $20.4 \pm 8.5$ & 19.3 & -1.92 & 0.06 \\
\hline \multicolumn{7}{|l|}{ NDSA } \\
\hline $\mathrm{SA}_{\mathrm{acc}}(n=12)$ & $0.37 \pm 0.28$ & 0.35 & $0.44 \pm 2.76$ & 0.29 & -0.24 & 0.14 \\
\hline $\mathrm{SA}_{\text {rot }}(n=12)$ & $1.20 \pm 1.20$ & 0.67 & $1.45 \pm 1.54$ & 0.91 & -1.02 & 0.31 \\
\hline $\operatorname{LDS}_{\mathrm{acc}}(n=12)$ & $1.55 \pm 0.93$ & 1.32 & $1.30 \pm 0.27$ & 1.34 & -0.08 & 0.94 \\
\hline $\operatorname{LDS}_{\text {rot }}(n=12)$ & $0.83 \pm 0.17$ & 0.81 & $0.85 \pm 0.26$ & 0.81 & -0.47 & 0.64 \\
\hline
\end{tabular}

Unit $\mathrm{cm}$

MFRT Modified Functional Reach Test, NDSA Nonlinear Dynamic Systems Analysis, SA spectral analysis, LDS local dynamic stability, acc anterior-posterior acceleration, rot angular acceleration around the mediolateral axis, $S D$ standard deviation

Table 3 The results of the open question

\begin{tabular}{lll}
\hline Answer & $\begin{array}{l}\text { Number of } \\
\text { subjects }\end{array}$ & $\begin{array}{l}\text { Percent of subjects } \\
n=13\end{array}$ \\
\hline $\begin{array}{l}\text { Better physical feeling } \\
\begin{array}{l}\text { Positive effect on the } \\
\text { psyche and morale }\end{array}\end{array}$ & III & 23.1 \\
$\begin{array}{l}\text { More force } \\
\text { More mobility }\end{array}$ & II & 7.7 \\
Less shoulder pain & II & 15.4 \\
Better trunk control & I & 15.4 \\
Continue the training & V & 7.7 \\
sessions & & 15.4 \\
\hline
\end{tabular}

and in clinical application, and is also scarcely used for wheelchair patients. Therefore, a comparison to other studies is difficult at this time.

SCIM III showed a $100 \%$ correlation between all three measurements in all subject. Only patients with a stable AIS score of at least 6 months were included in this study. A spontaneous recovery is thus largely unlikely and a chronic condition is assumed. The SCIM III is an instrument to record restrictions in the areas of self-care, breathing, bladder and bowel control as well as mobility [19]. In chronic SCI, the test for small changes in the subscores is far too unspecific to show changes which can be expected with curling training. Numerous prior studies that investigated SCIM III showed good sensitivity [20]. The difference is that in these studies testing was conducted in acute SCI during the rehabilitation and not in chronic SCI.

At the end of this study three of 13 participants described a subjective better physical feeling. Two subjects had the impression of having more mobility and two participants subjectively observed better trunk control. These statements indicate that wheelchair curling may be good preventive approaches to help persons with paraplegia prevent falling from the wheelchair. Better trunk control leads to a larger reaching movement and thus eases day-to-day life [6].

In the world of curling, a spirit of curling is mentioned, which is even described in the rules of the game [9]. This involves sportsmanship, team spirit, fairness and enjoyment of the game. Something of this spirit is also seen in the answers of the study participants to the open question at the end of the study. For example, one participant mentioned a positive effect on the psyche and morale, and three others had a better physical feeling in general. In this study there were no adverse events like injuries or falls from the wheelchair. In addition, the interest in this sport increased during the training sessions, which is reflected in the high attendance rate of $95 \%$. Five participants would very much have liked to continue the training sessions.

\section{Limitation}

A limiting factor for this study was the use of suitable assessments. The MFRT seems too unspecific for small progress in the therapy and the NDSA is still too little established for persons with SCI. In addition, the group of subjects of 13 persons might have been too small-despite the cross-over design-to present clearly the effect of wheelchair curling with the tests used.

\section{Conclusion}

The results of the curling training do indicate subjective improvement of the participants but do not show any significant effect on trunk control in persons with SCI. However, a tendency toward improvement is apparent. Future larger studies with more participants should follow up on this. No adverse events were reported. Thus, we believe indoor wheelchair curling training is safe physical activity 
for people with SCI. Wheelchair curling offers a suitable alternative to the types of sports already used in rehabilitation and in recreational opportunities for people with SCI. Participants subjectively described a change in their physical feeling. Above all, the increase in motivation, the positive effects on the psyche and the increased physical feeling of the subjects indicate that it would make sense to integrate wheelchair curling as a standard in the rehabilitation programme of paraplegics. It is a type of sport which can be played both with young and with old people and allows for a broad range of degrees of severity of SCI to be combined.

Acknowledgements The authors like to thank Dr. Philippe Terrier for his support in NDSA analysing.

\section{Compliance with ethical standards}

Conflict of interest The authors declare that they have no conflict of interest.

\section{References}

1. Lee BB, Cripps RA, Fitzharris M, Wing PC. The global map for traumatic spinal cord injury epidemiology: update 2011, global incidence rate. Spinal Cord. 2014;52:110-6.

2. Thietje R, Hirschfeld S. Epidemiology of spinal cord injury. In: Weidner N, Rupp R, Tansey KE, editors. Neurological aspects of spinal cord injury. 1st ed. Cham: Springer International Publishing; 2017. p. 3-17.

3. Zäch GA, Koch HG. Paraplegie: Ganzheitliche Rehabilitation. 1st ed. Basel: Karger; 2006.

4. Gauthier C, Gagnon D, Jacquemin G, Duclos C, Masani K, Popovic MR. Which trunk inclination directions best predict multidirectional-seated limits of stability among individuals with spinal cord injury? J Spinal Cord Med. 2012;35:343-50.

5. Hüter-Becker A, Dölken M. Physiotherapie in der Neurologie. 3rd ed. Stuttart: Georg Thieme Verlag; 2010.

6. Ditunno JF Jr., Young W, Donovan WH, Creasey G. The international standards booklet for neurological and functional classification of spinal cord injury. American Spinal Injury Association. Paraplegia . 1994;32:70-80.

7. Bjerkefors A, Thorstensson A. Effects of kayak ergometer training on motor performance in paraplegics. Int $\mathrm{J}$ Sports Med. 2006;27:824-9.

8. Boswell-Ruys CL, Sturnieks DL, Harvey LA, Sherrington C, Middleton JW, Lord SR. Validity and reliability of assessment tools for measuring unsupported sitting in people with a spinal cord injury. Arch Phys Med Rehabil. 2009;90:1571-7.

9. Federation WC. About wheelchair curling. Perth, Scotland: World Curling Federation; 2018. http://www.worldcurling.org/aboutwheelchair-curling.

10. Association BP. Wheelchair curling. London: British Paralympic Association; 2018. http://paralympics.org.uk/paralympicsports/w heelchair-curling.

11. Strubreither W, Neikes M, Stirnimann D, Eisenhuth J, Schulz B, Lude P. Klinische Psychologie bei Querschnittlähmung: Psychologische und psychotherapeutische Interventionen bei psychischen, somatischen und psychosozialen Folgen. 1st ed. Wien: Springer Verlag; 2014.

12. Schulgen G, Schumacher M. Cross-Over Studien. 1st ed. Berlin, Heidelberg: Springer Berlin Heidelberg; 2008. p. 317.

13. Federation WC. The rules of curling Perth. Scotland: World Curling Federation; 2018. http://www.worldcurling.org/rules-andregulations.

14. Lynch SM, Leahy P, Barker SP. Reliability of measurements obtained with a modified functional reach test in subjects with spinal cord injury. Phys Ther. 1998;78:128-33.

15. Granata KP, England SA. Stability of dynamic trunk movement Spine (Phila Pa 1976). 2006;31:E271-6.

16. Terrier P, Mittaz Hager AG, editors. A novel approach to asses the posturale control of unsupported sitting in patients with spinal cord injury: a feasibility study. In: 1st Clinical Movement Analysis World Conference. Rome; 2014.

17. Wirz M. Assessment: Spinal Cord Independence Measure (SCIM) - Die Selbstständigkeit erfassen. Physiopraxis. 2011;9:46-7.

18. Katz-Leurer M, Fisher I, Neeb M, Schwartz I, Carmeli E. Reliability and validity of the modified functional reach test at the subacute stage post-stroke. Disabil Rehabil. 2009;31:243-8.

19. Itzkovich M, Gelernter I, Biering-Sorensen F, Weeks C, Laramee MT, Craven BC, et al. The Spinal Cord Independence Measure (SCIM) version III: reliability and validity in a multi-center international study. Disabil Rehabil. 2007;29:1926-33.

20. Mehrholz J. Neuroreha nach Schlaganfall. Stuttgart: Georg Thieme Verlag; 2011. 